\title{
O papel da biopsia pulmonar na indicação cirúrgica de cardiopatias congênitas
}

Solange BORDIGNON*, Marizele E. MOLON *, Renato A. K. KALIL *, Fernando A. LUCCHESE *, Paulo R. PRATES *, Joāo Ricardo M. SANT'ANNA *, Ivo A. NESRALLA *

RBCCV 44205-187

BORDIGNON, S.; MOLON, M. E.; KALIL, R. A. K.; LUCCHESE, F. A.; PRATES, P. R.; SANT'ANNA, J. R. M.; NESRALLA, I. A. - O papel da biopsia pulmonar na indicação cirúrgica de cardiopatias congênitas. Rev. Bras. Cir. Cardiovasc., 7(4):256-262, 1992.

RESUMO: Objetivando relacionar o aspecto morfológico dos vasos pulmonares com os dados clínicos para auxiliar na decisão da cirurgia de cardiopatias congênitas com hipertensão pulmonar severa, em casos previamente avaliados por critérios clínicos, angiográficos e/ou hemodinâmicos, foram realizadas, de 1980 a 1991, no Instituto de Cardiologia do Rio Grande do Sul, 49 biopsias pulmonares. As idades dos pacientes variaram de $5 \mathrm{~m}$ a $28 \mathrm{a} 6 \mathrm{~m}$ (média $=7 \mathrm{a} 7 \mathrm{~m}$ ) e os diagnósticos foram: CIV (16), PCA (3), CIV e ESubAo (1), PCA e CIV (2), PCA e CIA (1) DSAV (7), DSAV incompl. e PCA (1), PCA e CoAo (1), Inter Arco Ao com PCA e CIV e/ou ESupraM e Anel SubAo (2), DVSVD com CIV e/ou PCA (6), DVSVE e EP (1), AT e ESubP. (1), TGV (1), TGV corrigida e CIV (1) e truncus arteriosus (5). Houve $3(6,1 \%)$ óbitos no pós-operatório da biopsia. Baseado na classificação de Heath-Edwards, foram para correçāo cirúrgica 11 pacientes com cardiopatias simples (CIV, PCA, DSAV), cuja mortalidade foi $36,4 \%$. Dez pacientes com cardiopatias complexas (DVSVD, DVSVE, TGV, truncus, inter arco Ao) foram para cirurgia, com mortalidade de $30 \%$. A evolução pós-operatória tardia foi favorável em $13(62 \%)$ pacientes. Conclui-se que a biopsia pulmonar pode ser útil na indicaçāo cirúrgica das cardiopatias congênitas com hipertensão pulmonar severa e, por envolver riscos, sua utilização deve ser criteriosa. É valiosa para os pacientes que apresentam dúvidas quanto ao grau de doença vascular pulmonar ou quanto à natureza das lesōes e o estudo clínico e hemodinâmico não são esclarecedores, ou mesmo quando apontarem para a contra-indicação cirúrgica.

DESCRITORES: biopsia pulmonar; hipertensāo arterial pulmonar.

\section{INTRODUÇĀO}

É de fundamental importância determinar o grau de doença vascular pulmonar associada a cardiopatias congênitas, para seu diagnóstico e prognóstico. A avaliaçāo clínica e o estudo hemodinâmico utilizados para graduar a resistência vascular pulmonar são relativamente precisos. Porém, em casos limítrofes, é necessário o auxílio de métodos mais objetivos, desde que a segurança diagnóstica é indispensável para a escolha do melhor tratamento aos pacientes nos quais a natureza, a causa e a severidade do comprometimento vascular pulmonar permanecem em dúvida. Nestes casos, a biopsia pulmonar para exame direto da morfologia dos vasos arteriais é utilizada ${ }^{7.9}$.

A biopsia pulmonar tem sido também empregada para estabelecer a conduta definitiva nos casos cuja hipertensão pulmonar parece claramente irreversível por outros critérios, visando à segurança na conduta indicada.

Este trabalho tem por objetivo relacionar os achados da biopsia pulmonar com condutas clínica

Trabalho realizado no Instituto de Cardiologia do Rio Grande do Sul. Fundaçáo Universitária de Cardiologia. Porto Alegre, RS, Brasil.

Apresentado ao $19^{\circ}$ Congresso Nacional de Cirurgia Cardíaca. São Paulo, SP, 7 a 9 de maio, 1992.

* Do Instituto de Cardiologia do Rio Grande do Sul.

Endereço para separatas: Solange Bordignon. Instituto de Cardiologia, Unidade de Pesquisa. Av. Princesa Izabel, 395,90620 Porto Alegre, RS, Brasil. 
BORDIGNON, S.; MOLON, M. E.; KALIL, R. A. K.; LUCCHESE, F. A.; PRATES, P. R.; SANT'ANNA, J. R. M.; NESRALLA, I. A. O papel da biopsia pulmonar na indicaçäo cirúrgica de cardiopatias congênitas. Rev. Bras. Cir. Cardiovasc., 7(4):256-262, 1992.

e cirúrgica, avaliando evolução, oportunidade e expectativa dos tratamentos indicados.

\section{CASUÍSTICA E MÉTODOS}

Foram analisadas as informaçōes de 49 pacientes com cardiopatia congênita submetidos a biopsia pulmonar. A idade variou dos 5 meses aos 28 anos e 6 meses (média de 7 anos e 7 meses). Vinte e seis eram do sexo masculino e todos de cor branca. Os diagnósticos constam da Tabela 1. A biopsia foi obtida por toracotomia ântero-lateral esquerda nos primeiros 7 pacientes, sendo retirado fragmento pulmonar, geralmente da língua, e, nos demais, por toracotomia ântero-lateral direita, sendo retirado um fragmento pulmonar da porção inferior do lobo superior direito. $O$ tamanho do fragmento foi proporcional ao paciente e sempre com mais de $1,5 \mathrm{~cm}$ de comprimento. O material foi inflado, fixado em formol a $10 \%$ e encaminhado para exame anatomopatológico. No período de pós-operatório foi mantido dreno de tórax por $24 \mathrm{~h}$, como rotina, e prestada assistência ventilatória conforme necessário, além dos cuidados rotineiros. Os pacientes receberam alta hospitalar no oitavo dia de pósoperatório.

O material para estudo histopatológico foi incluído em parafina. As coloraçōes utilizadas foram hematoxilina-eosina e Verhoett-Vam Gieson para

TABELA 1

BIOPSIA PULMONAR EM CARDIOPATIAS CONGENNITAS $\mathrm{n}=49$

Idade: $5 \mathrm{~m}$ a $28 \mathrm{a} 6 \mathrm{~m}$ (média=7a7m)

\begin{tabular}{lrr}
\hline LESĀO & $N$ & $\%$ \\
\hline - CIV & 16 & 32,7 \\
- CIV + ESubAo & 1 & 2,0 \\
- PCA + CIV & 2 & 4,1 \\
- PCA + CIA & 1 & 2,0 \\
- DSAV & 7 & 14,3 \\
- DSAV Inc+PCA & 1 & 2,0 \\
- Truncus Arteriosus & 5 & 10,0 \\
- TGV & 1 & 2,0 \\
- TGV Corrig + CIV & 1 & 2,0 \\
- AT + ESubP & 1 & 2,0 \\
- DSVE + EP & 1 & 2,0 \\
- DVSVD+CIV e/ou PCA & 6 & 12,0 \\
- PCA & 3 & 6,0 \\
- PCA + CoAo & 1 & 2,0 \\
- Inter Arco Ao+PCA+ & & \\
CIV e/ou ESupra M+ & & 4,1 \\
Anel SubAo & 2 & \\
\hline TOTAL & 49 & \\
\hline
\end{tabular}

(IC-FUC 1980-1991) fibras elásticas e colágeno. Neste estudo a lesão vascular foi avaliada quanto ao seu aspecto, severidade e o número e tamanho dos vasos afetados, de acordo com os critérios já descritos na literatura 1, 5, 6, 8.

Os critérios para indicação cirúrgica 9.14 foram baseados na avaliação da hipertensão pulmonar por estudos hemodinâmico, angiográfico e histológico, sendo que foram encaminhados para correçāo cirúrgica apenas aqueles pacientes que apresentavam grau histológico igual ou inferior a III na Classificação de Heath-Edwards. Os pacientes com grau III, nos quais o estudo morfométrico indicou grande extensão das lesōes, foram contra-indicados.

Os pacientes foram acompanhados nos ambulatórios, avaliados clinicamente em intervalos periódicos mensais e semestrais e submetidos a estudo ecocardiográfico uni e bidimensional, com Doppler; em $5(24 \%)$ pacientes foi realizado estudo hemodinâmico.

\section{RESULTADOS}

Para fins de análise, os pacientes foram separados em 2 grupos: pacientes com cardioplegia congênitas simples (persistência do canal arterial (PCA) - comunicação interventricular (CIV) - defeito septal atrioventricular (DSAV) e pacientes com cardiopatias congênitas complexas (interrupção do arco aórtico (Inter. Arco Ao) - truncus arteriousdupla via de saída de ventrículo direito (DVSVD) dupla via de saída de ventrículo esquerdo (DVSVE) - atresia pulmonar (AT) - transposição de grandes vasos (TGV) - transposição corrigida de grandes vasos (TGV corrigida e classificados histologicamente (Tabelas 2 e 3 ).

A mortalidade relacionada à biopsia pulmonar foi de $6,1 \%(n=3)$. Complicaçōes nâo-fatais da biopsia (Tabela 6) ocorreram em $7(14,1 \%)$ dos 49 casos, incluindo: 1 (2\%) caso de hemotórax, $1(2 \%)$

TABELA 2

CARDIOPATIAS CONGÊNITAS SIMPLES (PCA, CIV, DSAV)

\begin{tabular}{rc}
\hline GRAU DE HIPERTENSĀO PULMONAR & $N=32$ \\
\hline II & 2 \\
III & 6 \\
IV & 13 \\
Normal * & 8 \\
Nāo Avaliável & 1 \\
\hline
\end{tabular}

* Doença pulmonar, enfisema (IC-FUC 1980-1991) 
BORDIGNON, S.; MOLON, M. E.; KALIL, R. A. K.; LUCCHESE, F. A.; PRATES, P. R.; SANT'ANNA, J. R. M.; NESRALLA, I. A. O papel da biopsia pulmonar na indicação cirúrgica de cardiopatias congênitas. Rev. Bras. Cir. Cardiovasc., 7(4):256-262, 1992.

TABELA 3

CARDIOPATIAS CONGENNITAS COMPLEXAS (INTER. ARCO AO TRUNCUS DVSVD DVSVE AT TGV)

\begin{tabular}{cc}
\hline GRAU DE HIPERTENSÃO PULMONAR & N=17 \\
\hline II & 4 \\
II & 6 \\
III & 4 \\
IV & 3 \\
\hline
\end{tabular}

(IC-FUC 1980-1991)

caso de pneumonia, 1 (2\%) caso de deiscência de incisāo, $1(2 \%)$ caso de infecçāo local, $2(4,1 \%)$ casos de reoperação por sangramento e 1 (2\%) caso de encefalopatia anóxica.

Dos pacientes portadores de CIV $(n=16), 6$ $(37,5 \%)$ foram encaminhados a cirurgia, sem mortalidade pós-operatória. Como complicaçōes nãofatais, $3(50 \%)$ pacientes apresentaram CIV residual mínima, $1(16,7 \%)$ apresentou hiperternsāo pulmonar progressiva, $1(16,7 \%)$ BAV total, $1(16,7 \%)$ caso de ritmo juncional e $2(33,3 \%)$ pacientes apresentaram insuficiência ventilatória com tempo prolongado de ventilaçāo mecânica, além de infecçāo respiratória e dano neurológico transitório.

Dois pacientes portadores de PCA foram a correçāo cirúrgica, com mortalidade de $50 \% \quad(n=1)$, sendo que 1 caso de PCA e CoAo levado a cirurgia também foi a êxito letal $(100 \%)$.

Dois pacientes com DSAV foram operados e faleceram.

Nos pacientes com DVSVD com CIV/PCA operados $(n=3)$, houve mortalidade de $66,6 \% \quad(n=2)$, sendo que $1(33,3 \%)$ paciente teve como complica- çāo nāo-fatal CIV residual mínima. Um paciente com DVSVE foi operado e apresentou, na evolução pós-operatória, sepsis e insuficiência renal aguda. Dois pacientes com TGV foram operados, sendo que $1(50 \%)$ apresentou infecção respiratória e o outro $(50 \%)$ apresentou BAV transitório e CIV residual.

Dos pacientes portadores de truncus arteriosus, $2(40 \%)$ foram a cirurgia cardíaca, sendo que a mortalidade pós-operatória foi de $50 \% \quad(n=1)$.

Dois pacientes com Interrupção de arco Ao foram a cirurgia, sendo que $1(50 \%)$ evoluiu com insuficiência cardíaca congestiva no pós-operatório imediato (Tabelas 4 e 5).

Dos 49 pacientes submetidos a biopsia pulmonar no Instituto de Cardiologia do Rio Grande do Sul, $10(20,4 \%)$ foram encaminhados a tratamento clínico por apresentarem lesōes plexiformes (grau IV de Heath - Edwards), com uma sobrevida média de 1 ano e 5 meses. Destes, $2(4,1 \%)$ morreram em decorrência da biopsia pulmonar. Um (2\%) paciente portador de lesōes plexiformes foi submetido a cirurgia de Mustard paliativa com boa evolução pósoperatória em 5 meses de seguimento.

Nos restantes $35(71,4 \%)$ pacientes com lesōes iguais ou menores que III de Health-Edwards (H-E), $21(60 \%)$ pacientes foram encaminhados a cirurgia corretiva, ocorrendo $7(33,3 \%)$ óbitos no pós-operatório e $1(4,8 \%)$ apresentou hipertensão pulmonar progressiva no pós-operatório tardio.

Dos $14(40 \%)$ pacientes submetidos a tratamento clínico, houve $1(2,9 \%)$ óbito pós-biopsia e os demais $13(37,1 \%)$ pacientes apresentaram boa sobrevida pós biopsia pulmonar, com média de 1 ano e 7 meses de acompanhamento ambulatorial.

TABELA 4

CARDIOPATIAS CONGÊNITAS SIMPLES CORREÇĀO CIRÚRGICA

\begin{tabular}{|c|c|c|c|c|c|c|}
\hline \multirow[t]{2}{*}{ LESĀO } & \multirow{2}{*}{$\begin{array}{c}\text { CASOS OPERADOS } \\
N\end{array}$} & \multicolumn{2}{|c|}{ MORTALIDADE } & \multirow{2}{*}{$\begin{array}{c}\text { COMPLICAÇŌES } \\
\text { NĀO-FATAIS }\end{array}$} & \multirow[b]{2}{*}{$N$} & \multirow[b]{2}{*}{$\%$} \\
\hline & & $N$ & $\%$ & & & \\
\hline - CIV & 6 & 0 & 0 & \multirow{6}{*}{$\begin{array}{l}\text { - CIV Residual } \\
\text { - HP Progres. } \\
\text { - BAV Total } \\
\text { - R. Juncional } \\
\text { - Insuf. Vent.+ Infecção+Dano } \\
\text { Neurológico Transitório }\end{array}$} & \multirow{3}{*}{$\begin{array}{l}3 \\
1 \\
1 \\
1\end{array}$} & \multirow{3}{*}{$\begin{array}{l}50 \\
16,7 \\
16,7 \\
16,7 \\
33,3\end{array}$} \\
\hline & & & & & & \\
\hline & & & & & & \\
\hline - PCA & 2 & 1 & 50 & & & \\
\hline - $\mathrm{PCA}+\mathrm{CoAO}$ & 1 & 1 & 100 & & & \\
\hline - DSAV & 2 & 2 & 100 & & & \\
\hline TOTAL & 11 & 4 & 36,4 & & & \\
\hline
\end{tabular}


BORDIGNON, S.; MOLON, M. E.; KALIL, R. A. K.; LUCCHESE, F. A.; PRATES, P. R.; SANT'ANNA, J. R. M.; NESRALLA, I. A. O papel da biopsia pulmonar na indicaçāo cirúrgica de cardiopatias congênitas. Rev. Bras. Cir. Cardiovasc., 7(4):256-262, 1992.

TABELA 5

CARDIOPATIAS CONGÊNITAS COMPLEXAS - CORREÇÃO CIRÚRGICA

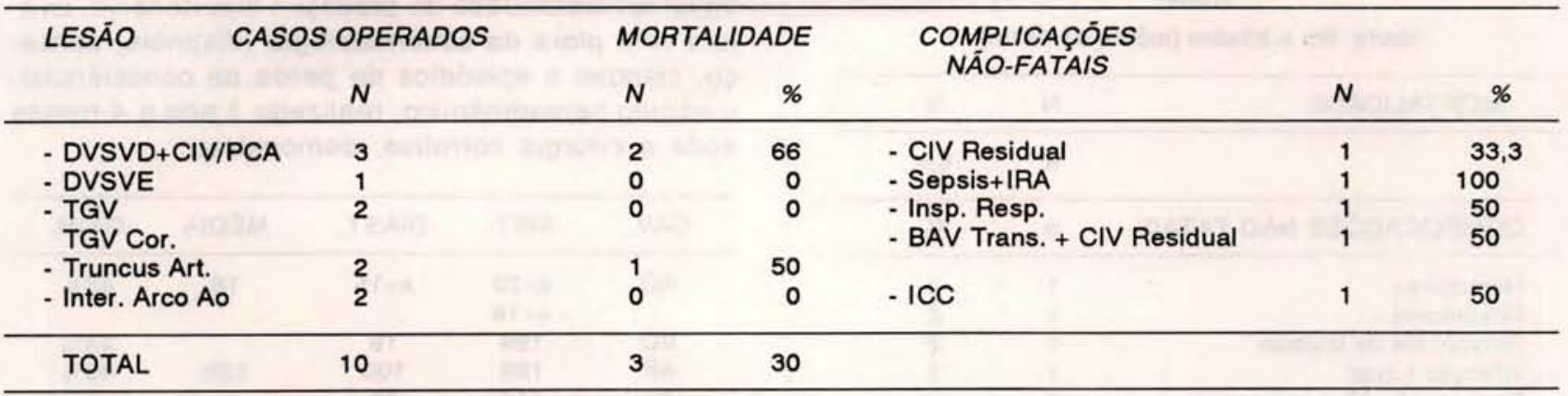

(IC-FUC 1980-1991)

Em $2(4,1 \%)$ pacientes nāo foi possível avaliar - grau de hipertensão pulmonar por problemas técnicos; levados a cirurgia corretiva, apresentaram boa evolução pós-operatória, num seguimento de 1 ano e 1 mês.
Um (2\%) paciente com circulação pulmonar normal foi encaminhando a cirurgia corretiva e evoluiu bem, em 1 ano e 7 meses de acompanhamento clínico ambulatorial (Quadro 1).

Para fins de ilustraçāo, descrevemos, a seguir,

QUADRO 1

EVOLUÇĀO DOS PACIENTES SUBMETIDOS A BIOPSIA PULMONAR NO IC-FUC JANEIRO DE 1980 A DEZEMBRO DE 1991.
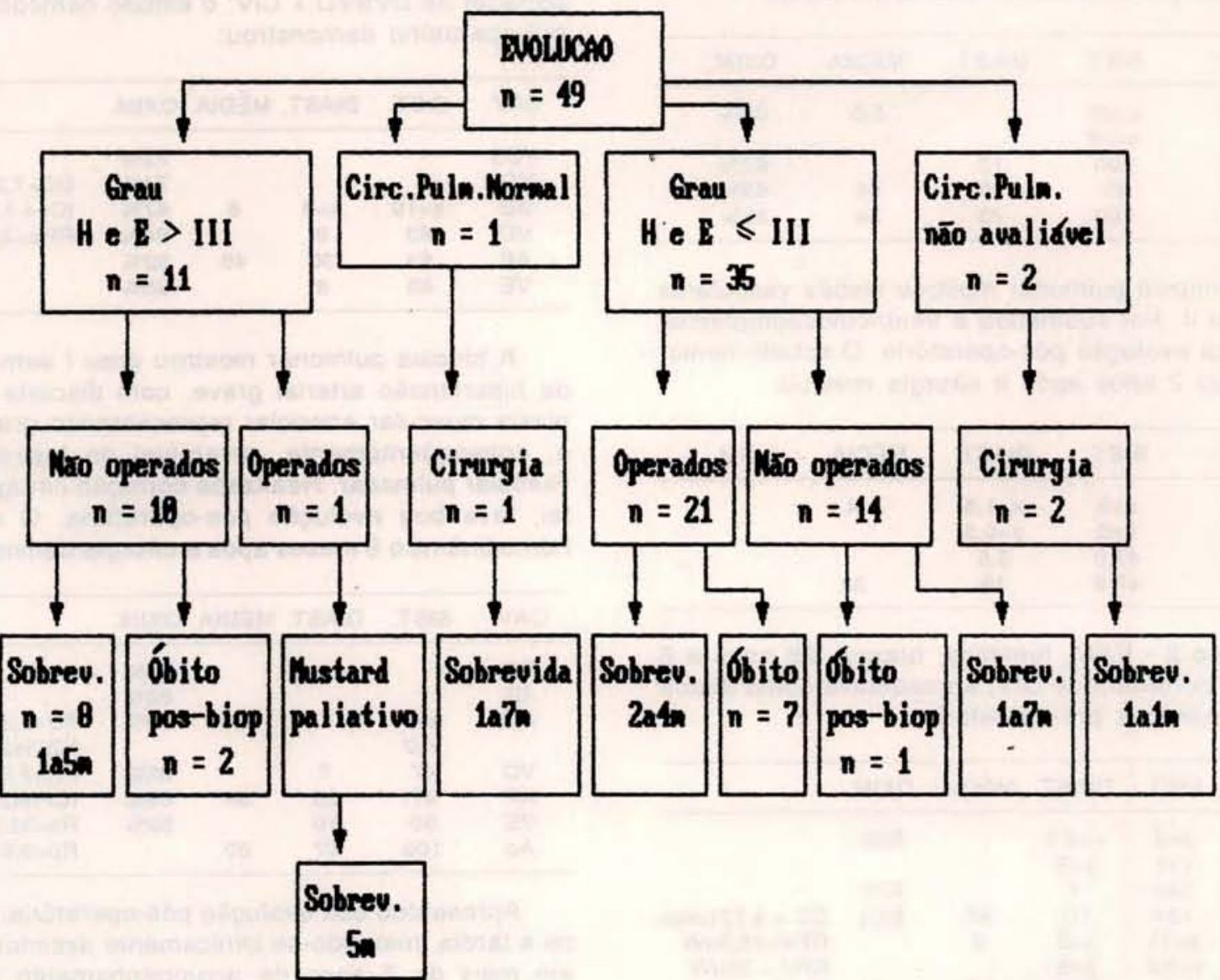
BORDIGNON, S.; MOLON, M. E.; KALIL, R. A. K.; LUCCHESE, F. A.; PRATES, P. R.; SANT'ANNA, J. R. M.; NESRALLA, I. A. O papel da biopsia pulmonar na indicaçāo cirúrgica de cardiopatias congênitas. Rev. Bras. Cir. Cardiovasc., 7(4):256-262, 1992.

\section{TABELA 6 \\ BIÓPSIA PULMONAR EM CARDIOPATIAS CONGÊNITAS $\mathrm{N}=49$ \\ Idade: $5 \mathrm{~m}$ a $28 \mathrm{a} 6 \mathrm{~m}$ (média de $7 \mathrm{a} 7 \mathrm{~m}$ )}

\begin{tabular}{lcc}
\hline MORTALIDADE & $\mathrm{N}$ & $\%$ \\
\hline & 3 & 6,1 \\
\hline COMPLICAÇŌES NÃO-FATAIS & $\mathrm{n}$ & $\%$ \\
\hline - Hemotórax & 1 & 2 \\
- Pneumonia & 1 & 2 \\
- Deiscência de Incisāo & 1 & 2 \\
- Infecção Local & 1 & 2 \\
- Reoperaçāo (Sangramento) & 2 & 4,1 \\
- Encefalopatia Anóxica & 1 & 2 \\
\hline
\end{tabular}

(IC-FUC 1980-1991)

3 casos de pacientes cirúrgicos que apresentaram na sua evolução comportamentos diversos.

Caso 1 - CIF, 12 anos, feminina, branca com diagnóstico de CIV; submetida a estudo hemodinâmico pré-operatório, que demonstrou:

\begin{tabular}{ccccc}
\hline CAV. & SIST. & DIAST. & MÉDIA & OXIM. \\
\hline AD & $a=20$ & & 5,5 & $63 \%$ \\
VD & $v=16$ & & & \\
AP & 100 & 12 & & $67 \%$ \\
Ao & 100 & 56 & 65 & $65 \%$ \\
\hline
\end{tabular}

A biopsia pulmonar mostrou lesōes vasculares de grau II. Foi submetida a ventriculoseptoplastia, com boa evolução pós-operatória. $O$ estudo hemodinâmico 2 anos após a cirurgia revelou:

\begin{tabular}{|c|c|c|c|c|}
\hline CAV. & SIST. & DIAST. & MÉDIA & OXIM. \\
\hline$A D$ & $\begin{array}{l}a=6 \\
v=5\end{array}$ & $\begin{array}{l}x=1,5 \\
y=0,5\end{array}$ & 4 & \\
\hline VD & 47,5 & 3,5 & & \\
\hline AP & 47,5 & 19 & 32 & \\
\hline
\end{tabular}

Caso 2 - ESM, feminina, branca, 28 anos e 6 meses, portadora de CIV, apresentava como dados hemodinâmicos pré-operatórios:

\begin{tabular}{|c|c|c|c|c|c|}
\hline CAV. & SIST. & DIAST. & MÉDIA & OXIM. & \\
\hline$A D$ & $\begin{array}{l}a=4 \\
v=1\end{array}$ & $\begin{array}{c}x=3,5 \\
y=3\end{array}$ & & $53 \%$ & \\
\hline VD & 134 & 4 & & $57 \%$ & \\
\hline $\begin{array}{l}\mathrm{AP} \\
\mathrm{CP}\end{array}$ & $\begin{array}{c}134 \\
a=11\end{array}$ & $\begin{array}{c}60 \\
x=9\end{array}$ & $\begin{array}{c}96 \\
9\end{array}$ & $63 \%$ & $\begin{array}{l}\mathrm{DC}=4,721 / \mathrm{min} \\
\mathrm{RPV}=18,5 \mathrm{uW}\end{array}$ \\
\hline & $v=9,5$ & $y=8$ & & & $\mathrm{RPV}=21 \mathrm{uW}$ \\
\hline Ao & 136 & 80 & 100 & $78 \%$ & $\mathrm{Rp} / \mathrm{Rs}=0,9$ \\
\hline
\end{tabular}

A biópsia pulmonar mostrou grau III, sendo que o aspecto das alteraçōes vasculares sugeriam provável reversibilidade do processo hipertensivo. Evoluiu com piora da sintomatologia (dispnéia, cansaço, cianose e episódios de perda da consciência); o estudo hemodinâmico, realizado 1 ano e 4 meses após a cirurgia corretiva, demonstrou:

\begin{tabular}{|c|c|c|c|c|}
\hline CAV. & SIST. & DIAST. & MÉDIA & OXIM. \\
\hline$A D$ & $\begin{array}{l}a=20 \\
v=19\end{array}$ & $x=15$ & 16 & $45 \%$ \\
\hline $\begin{array}{l}\text { VD } \\
\text { AP }\end{array}$ & $\begin{array}{l}198 \\
198\end{array}$ & $\begin{array}{c}19 \\
100\end{array}$ & 126 & $\begin{array}{l}45 \% \\
43 \%\end{array}$ \\
\hline Ao & 110 & 90 & & \\
\hline
\end{tabular}

Pressões em TAP após uso de nifedipina SL:

$\begin{array}{lll}S=200 \mathrm{mmHg} & D=104 \mathrm{mmHg} & M=132 \mathrm{mmHg} \\ \text { Ao } S=140 \mathrm{mmHg} & D=100 \mathrm{mmHg}\end{array}$

Foi iniciado tratamento com antagonista do cálcio, $30 \mathrm{mg} /$ dia com discreta melhora do quadro clínico.

Caso 3 - ASHF, 9 anos, masculino, branco, portador de DVSVD + CIV; o estudo hemodinâmico pré-operatório demonstrou:

\begin{tabular}{|c|c|c|c|c|c|}
\hline CAV. & SIST. & DIAST. & IÉDIA & OXIM. & \\
\hline VCs & & & & $72 \%$ & \\
\hline $\mathrm{VCl}$ & & & & $74 \%$ & $\mathrm{DC}=7,21 / \mathrm{min}$ \\
\hline$A D$ & $a=10$ & $x=3$ & 6 & $67 \%$ & $\mathrm{IC}=4 \mathrm{1} / \mathrm{min}$ \\
\hline VD & 63 & 9 & & $84 \%$ & $\mathrm{RPp}=2,4 \mathrm{uW}$ \\
\hline AP & 63 & 30 & 46 & $92 \%$ & \\
\hline VE & 63 & 6 & & $95 \%$ & \\
\hline
\end{tabular}

A biopsia pulmonar mostrou grau I sem sinais de hipertensão arterial grave, com discreta hiperplasia muscular arteriolar representando grau leve e, conseqüentemente, reversível de hipertensāo vascular pulmonar. Realizada correção cirúrgica total, teve boa evolução pós-operatória. $O$ estudo hemodinâmico 8 meses após a cirurgia demonstrou:

\begin{tabular}{|c|c|c|c|c|c|}
\hline CAV. & SIST. & DIAST. & MÉDIA & OXIM. & \\
\hline $\begin{array}{l}\mathrm{CS} \\
\mathrm{Cl}\end{array}$ & & & & $\begin{array}{l}66 \% \\
66 \%\end{array}$ & \\
\hline$A D$ & $\begin{array}{l}a=8 \\
v=7\end{array}$ & & 5,5 & $60 \%$ & $\begin{array}{l}\mathrm{FS}=2,31 / \mathrm{min} \\
\mathrm{ICS} / \mathrm{m} 2=2,7\end{array}$ \\
\hline VD & 47 & 7 & & $65 \%$ & $\mathrm{FP}=2,5 \mathrm{1} / \mathrm{min}$ \\
\hline AP & 47 & 20 & 34 & $68 \%$ & $1 \mathrm{CP} / \mathrm{m} 2=2,9$ \\
\hline VE & 98 & 10 & & $89 \%$ & $R s=32 u W$ \\
\hline Ao & 100 & 67 & 80 & & $R p=9,6 u W$ \\
\hline
\end{tabular}

Apresentou boa evolução pós-operatória precoce e tardia, matendo-se clinicamente assintomático em mais de 7 anos de acompanhamento ambulatorial. 
BORDIGNON, S.; MOLON, M. E.; KALIL, R. A. K.; LUCCHESE, F. A.; PRATES, P. R.; SANT'ANNA, J. R. M.; NESRALLA, I. A. O papel da biopsia pulmonar na indicaçāo cirúrgica de cardiopatias congênitas. Rev. Bras. Cir. Cardiovasc., 7(4):256-262, 1992.

\section{COMENTÁRIOS}

Os clássicos trabalhos de HEATH et alii ${ }^{3} \mathrm{e}$ HEATH \& EDWARDS ${ }^{2}$ descreveram a doença vascular pulmonar por hipertensāo, procurando correlacioná-la com os dados de hemodinâmica e tentando entender a evolução das lesōes vasculares pulmonares secundárias e cardiopatias congênitas. Os estudos mais recentes ${ }^{5,7,10}$ utilizando critérios de análise morfométrica correlacionados com os dados hemodinâmicos permitiram uma análise bem mais precisa das alteraçōes vasculares pulmonares. O estudo histopatológico dos vasos pulmonares tem sido utilizado como auxiliar na decisão cirúrgica para escolha do procedimento mais adequado em cada caso ${ }^{9} .10$, baseando-se na previsão cirúrgica (correçāo precoce no primeiro ou segundo ano de vida, conforme a doença e operabilidade (conforme avaliação adequada e mortalidade cirúrgica, levando em conta que não há limite claro entre operabilidade ou nāo). Espera-se que pacientes com menos de 2 anos apresentem reversibilidade das alteraçōes vasculares ${ }^{4,7.14} \mathrm{e}$ que dos 2 anos aos 5 anos possam se beneficiar com o tratamento cirúrgico, conforme o tipo de correçăo e o grau de hipertensão, sendo o prognóstico determinado pela doença, pela idade, grau de hipertensāo pulmonar e tipo de correção.
Em nossa avaliação, o fato de todos os pacientes apresentarem graus avançados de hipertensāo arterial pulmonar e, conseqüentemente, dúvida quanto à sua possível reversibilidade pelos dados clínicos, hemodinâmicos e angiográficos, nos levou a optar pela biopsia pulmonar, a qual nos permitiu decidir por cirurgia corretiva em $21(42,9 \%)$ pacientes sendo que, destes, apenas $7(33 \%)$ evoluíram para o óbito no pós-operatório.

Levando-me em consideração que nestes pacientes a conduta clássica seria a contra-indicaçāo de cirurgia corretiva, devido ao elevado grau de repercussão da hipertensão pulmonar, conclui-se que a biópsia pulmonar pode ser útil na indicaçāo cirúrgica das cardiopatias congênitas; apesar dos riscos que the são inerentes, é valiosa para pacientes nos quais seja necessário ter segurança quanto ao grau de doença pulmonar ou à natureza das lesōes. Da mesma forma, quando o estudo clínico e o hemodinâmico nāo forem suficientemente esclarecedores, ou ainda, quando os demais critérios apontam claramente para a contra-indicação de cirurgia corretiva, a biopsia torna-se mais valiosa. Nestes casos, ficou evidente, pela evolução dos pacientes, que a biópsia deve ser o único critério para selar definitivamente o prognóstico do paciente.

BORDIGNON, S.; MOLON, M. E.; KALIL, R. A. K.; LUCCHESE, F. A.; PRATES, P. R.; SANT'ANNA, J. R. M.; NESRALLA, I. A. - The role of lung biopsy in the decision-making process for congenital heart disease. Rev. Bras. Cir. Cardiovasc., 7(4):256-262, 1992.

ABSTRACT: Open lung biopsy has been performed in patients with congenital heart disease and severe pulmonary hypertension in order to complement the decision-making process for surgical correction. All patients had clinical, hemodynamic and angiographic evaluation previously and would not be surgical candidates by conventional criteria. The 49 patients were studied from 1980 to 1991 at the Instituto de Cardiologia do Rio Grande do Sul, Brazil. Age range was $5 \mathrm{~m}$ to $28 \mathrm{y} 6 \mathrm{~m}$ (mean=7y7m). The diagnoses were: VSD (16); PDA (3); VSD and SubAortic Stenosis (1); PDA and VSD (2); PDA and ASD (1); AVSD (7); Incomplete AVSV and PDA (1); PDA and ACo (1); AAI with PDA and VSD (2); DORV with VSD and or PDA (6); DOLV with PS (1); TA (1), TGA (1); Corrected TGA with VSD (1) and Truncus Arteriosus (5). There were 3 hospitalar deaths after the biopsy procedure $(6.1 \%)$. When the pulmonary vascular lesions were classified as Heath-Edwards class III or less, surgical correction was performed. There were 21 patients, 11 simple defects (PDA; VSD; AVSD) with 4 deaths $(36.4 \%)$ and 10 complex heart defects with 3 deaths $(30 \%)$. One patient with VSD had persistent evolutive pulmonary hypertension. The remaining 13 had benefited from the correction. In conclusion, lung biopsy may be useful for the surgical decision in congenital heart disease with severe pulmonary hypertension. As a surgical procedure with the involved risks, it must be selectively indicated. Most patients with vascular lesions below class III ( $\mathrm{H}-\mathrm{E})$, despite other methods of evaluation would benefit from the corrective procedure.

DESCRIPTORS: hypertension, pulmonary; lung biopsy. 
BORDIGNON, S.; MOLON, M. E.; KALIL, R. A. K.; LUCCHESE, F. A.; PRATES, P. R.; SANT'ANNA, J. R. M.; NESRALLA, I. A. O papel da biopsia pulmonar na indicaçāo cirúrgica de cardiopatias congênitas. Rev. Bras. Cir. Cardiovasc., 7(4):256-262, 1992.

AGRADECIMENTO: Agradecemos à Dra. Lígia Coutinho e à Dra. Marinêz Barra Rossi, pela realização dos exames anatomopatológicos.

\section{REFERÊNCIAS BIBLIOGRÁFICAS}

1 FRIED, R.; FALKOVSKI, G.; NEWBURGER, J.; GORCHAKOVA, A. I.; RABINOVITCH, M.; GORDONOVA, M. I.; FYLER, D.; REID, L.; BURAKOVSKY, V. - Pulmonary arterial changes in patients with ventricular septal defects and severe pulmonary hipertension. Ped. Cardiol., 7: 147-154, 1986.

HEATH, D. \& EDWARDS, J. E. - The pathology of hypertensive pulmonary vascular disease: a description of six grades of structural changes in the pulmonary arteries with special reference to congenital cardiac septal defects. Circulation, 18: 533-547, 1958.

HEATH, D.; HELMOORTZ Jr., H. F.; BURCHELL, H. B.; DUSHANE, J. W.; EDWARDS, J. E. - Graded pulmonary vascular changes and hemodynamic findings in cases ao atrial and ventricular septal defect and patent ductus arterious. Circulation, 18: 1155 1166, 1958.

4 HEATH, D.; HELMHOLZ Jr., H. F.; BURCHELL, H. B.; DUSHANE, J. W.; KIRKLIN, J. W.; EDWARDS, J. E. - Relation between structural changes in the small pulmonary arteries and the immediate reversibility of pulmonary hypertension following closure of ventricular and atrial septal defect. Circulation, 1167-1174, 1958.

HAWORTH, S. G. - Understanding pulmonary vascular disease in young children. Internat. J. Cardiol., 15: 101-103, 1987.

6

HAWORTH, S. G.; RADLEY-SMITH, R.; YACOUB, M. - Lung biopsy findings in transposition of the great arteries with ventricular septal defect: potentially reverseble pulmonary vascular disease is not always synonymous with operability.

J. Am. College Cardiol., 9: 327-333, 1987.

7 HAWORTH, S. G. \& REID, L. - A morphometric study of regional variation in lung structure in infants with pulmonary hypertension and congenital cardiac defect a justification of lung piospy. Br. Heart J., 40: 825831, 1978.

8 KALIL, R. A. K.; COUTINHO, L.; LUCCHESE, F. A.; PRATES, P. R.; SANT'ANNA, J. R.; PEREIRA, E.; NESRALLA, I. A.; CARDOSO, C. R. - Biópsia pulmonar para avaliaçāo da doença vascular secundária às cardiopatias congênitas. Arq. Bras. Cardiol., 41: 199-204, 1983.

9 MARCELLETTI, G.; WAGENVOORT, C. A.; LOSE KOOT, T. G.; BECKER, A. E. - Paliative Mustard or Rastelli operation in complete transportation of the great arteries: option decided by lung biopsy. J. Thorac. Cardiovasc. Surg., 77: 677-682, 1979.

10 RABINOVITCH, M.; CASTANEDA, A. R.; REID, L. Lung biopsy with frozen section as a diagnostic aid in patients with congenital heart defects. Am. J. Cardiol., 47: 77-83, 1981.

11 WAGENVOORT, C. A. - Lung biopsy specimens in the evaluation of pulmonary vascular disease. Chest, 77: 614-621, 1980.

12 WAGENVOORT, C. A.; NAUTA, J.; VAN DER SCHAAR, P. J.; WEEDA, H. W. H.; WAGENVOORT, N. - Effect of flow and pressure on pulmonary vessels: semi quantitative study based on lung biopsies. Circulation, 25: 1028-1037, 1967.

13 WILSON, N. J.; SEAR, M. D.; TAYLOR, G. P.; LEBLANC, J. E.; SANDOR, G. G. S. - The clinical valve and risks of lung biopsy in children with congenital heart disease. J. Thorac. Cardiovasc. Surg., 99: 460-468, 1990.

14 YAMAKI, S.; MOHRI, H.; HANEDA, K.; ENDO, M.; AKIMOTO, H. - Indications for surgery based on lung biopsy in cases of ventricular septal defect and/ or patent ductus arteriosus with severe pulmonary hypertension. Chest, 96: 31-39, 1989. 